\title{
Epidemiological characteristics of bacillary dysentery from 2009 to 2016 and its incidence prediction model based on meteorological factors
}

\author{
Qiuyu Meng ${ }^{1+}$, Xun Liu ${ }^{2 \dagger}$, Jiajia Xie ${ }^{1}$, Dayong Xiao ${ }^{3}, Y_{i}$ Wang $^{1}$ and Dan Deng ${ }^{1 *}$ (D)
}

\begin{abstract}
Background: This study aimed to analyse the epidemiological characteristics of bacillary dysentery (BD) caused by Shigella in Chongqing, China, and to establish incidence prediction models based on the correlation between meteorological factors and BD, thus providing a scientific basis for the prevention and control of BD.

Methods: In this study, descriptive methods were employed to investigate the epidemiological distribution of BD. The Boruta algorithm was used to estimate the correlation between meteorological factors and BD incidence. The genetic algorithm (GA) combined with support vector regression (SVR) was used to establish the prediction models for $\mathrm{BD}$ incidence.

Results: In total, 68,855 cases of BD were included. The incidence declined from 36.312/100,000 to 23.613/100,000, with an obvious seasonal peak from May to October. Males were more predisposed to the infection than females (the ratio was 1.118:1). Children < 5 years old comprised the highest incidence $(295.892 / 100,000)$ among all age categories, and pre-education children comprised the highest proportion (34,658 cases, 50.335\%) among all occupational categories. Eight important meteorological factors, including the highest temperature, average temperature, average air pressure, precipitation and sunshine, were correlated with the monthly incidence of BD. The obtained mean absolute percent error (MAPE), mean squared error (MSE) and squared correlation coefficient $\left(R^{2}\right)$ of GA_SVR_MONTH values were 0.087, 0.101 and 0.922 , respectively.

Conclusion: From 2009 to 2016, BD incidence in Chongqing was still high, especially in the main urban areas and among the male and pre-education children populations. Eight meteorological factors, including temperature, air pressure, precipitation and sunshine, were the most important correlative feature sets of BD incidence. Moreover, $\mathrm{BD}$ incidence prediction models based on meteorological factors had better prediction accuracies. The findings in this study could provide a panorama of BD in Chongqing and offer a useful approach for predicting the incidence of infectious disease. Furthermore, this information could be used to improve current interventions and public health planning.
\end{abstract}

Keywords: Shigella, Dysentery, Meteorological factors, Boruta algorithm, Genetic algorithm, Support vector regression, Predictive model, China

\footnotetext{
* Correspondence: 100079@cqmu.edu.cn

${ }^{\dagger}$ Qiuyu Meng and Xun Liu contributed equally to this study and should be considered co-first authors

${ }^{1}$ School of Public Health and Management, Research Center for Medicine and Social Development, Innovation Center for Social Risk Governance in Health, Chongqing Medical University, Chongqing 400016, China

Full list of author information is available at the end of the article
}

(c) The Author(s). 2019 Open Access This article is distributed under the terms of the Creative Commons Attribution 4.0 International License (http://creativecommons.org/licenses/by/4.0/), which permits unrestricted use, distribution, and reproduction in any medium, provided you give appropriate credit to the original author(s) and the source, provide a link to the Creative Commons license, and indicate if changes were made. The Creative Commons Public Domain Dedication waiver (http://creativecommons.org/publicdomain/zero/1.0/) applies to the data made available in this article, unless otherwise stated. 


\section{Background}

Bacillary dysentery (BD) is a serious infectious intestinal disease caused by Shigella. The disease is transmitted via the oral-faecal route or through contact with contaminated water and food. The main clinical manifestations of $\mathrm{BD}$ are diarrhoea, fever, abdominal colic, etc. [1, 2]. $\mathrm{BD}$ is one of the most common causes of diarrhoea. Christopher et al. estimated that Shigella infection is the second leading cause of diarrhoeal death, with approximately 164,300 deaths caused by BD worldwide in 2015 . Of these deaths, 54,900 were of children under the age of 5 , accounting for $12.5 \%$ of the total [3]. In China, BD imposes a considerable public health burden; nearly 123 , 283 cases of bacillary and amoebic dysentery occur on an annual basis, placing this disease within the top five infectious diseases in China in 2016 [4].

Clearly, the harmful effects of $\mathrm{BD}$ on humans cannot be ignored. We urgently need to find effective prevention and control measures. However, the risk of infectious diseases has always been affected by multiple factors, such as climate, the regional economy and environmental health. The impacts of meteorological factors on diseases, especially infectious diseases, are of particular current concern to researchers, and relevant studies have been comprehensive [5-10]. Numerous studies have concluded that BD transmission might be influenced by meteorological factors [11-14]. For example, a study in Chaoyang District, Beijing, used a structural equation model (SEM) to analyse the correlation between meteorological factors and BD incidence and showed that $\mathrm{BD}$ incidence was positively correlated with air temperature and negatively correlated with sunshine [11]. A study in Hunan Province, China, used a Bayesian space-time hierarchical model (BSTHM) to analyse the effect of meteorological factors on BD and found that BD incidence increased by $3.194 \%$ with every $1{ }^{\circ} \mathrm{C}$ rise in temperature and increased by $0.674 \%$ with every $1 \%$ increase in relative humidity [12]. A study in Jinan City, China, adopted a distributed lag nonlinear model (DLNM) to analyse the lagged effect of meteorological factors on $\mathrm{BD}$ incidence and showed that each $5{ }^{\circ} \mathrm{C}$ rise in temperature increased the number of BD cases by $61 \%$ at lag 0-lag 7 days [13]. Similarly, a study in Hefei Province, China, adopted the DLNM model to analyse the lagged effect of meteorological factors on BD. The authors found that the risk of $\mathrm{BD}$ increased with the temperature rise above a threshold $\left(18.4{ }^{\circ} \mathrm{C}\right)$, and the temperature effects appeared to be acute; the proportion of BD attributable to hot temperatures (temperature $=31.2^{\circ} \mathrm{C}$ ) was $18.74 \%$ [14].

While there are many studies on the correlation between $\mathrm{BD}$ and meteorological factors, researchers have neglected another important issue, which is how to use the correlation between $\mathrm{BD}$ and meteorological factors to establish a prediction model of $\mathrm{BD}$ incidence. The prediction of the incidence of infectious diseases is the basis of epidemic prevention and control. It can provide decision-making support for relevant health departments to formulate solutions and reduce risk and loss, and it is of great significance in the field of disease research and the formulation of epidemic prevention and control strategies.

According to a previous study, BD incidence in some areas of Chongqing has been relatively high in recent years [15]. This suggests that it is necessary to investigate the current BD situation throughout Chongqing. Therefore, this paper consists of two parts. First, an epidemiological description of current situation of the BD epidemic in Chongqing from 2009 to 2016 is provided to demonstrate the spatial, temporal and population distribution of this epidemic. Second, based on the correlation between meteorological factors and $\mathrm{BD}$ incidence, prediction models for $\mathrm{BD}$ incidence are established, and the role of meteorological factors in improving model accuracy is discussed. The latter part is our key research content.

\section{Materials and methods Survey region}

Located in the southwestern part of inland China, Chongqing is the only municipality directly under the control of the central government in western China. Its longitude is $105^{\circ} 11^{\prime} \sim 110^{\circ} 11^{\prime}$, and its latitude is $28^{\circ} 10^{\prime}$ $\sim 32^{\circ} 13^{\prime}$ (Fig. 1). Chongqing's landforms are dominated by hills and mountains, which account for $76 \%$ of the area. The city has a total population of approximately 30.75 million over an area of $82,403.10 \mathrm{~km}^{2}$ and administers 38 counties. Chongqing has a continental subtropical monsoon humid climate, with four distinctive seasons: a cold winter, a hot summer, a spring with variable temperatures and a fall during which temperatures drop steeply. Rain is concentrated during the summer and autumn. The annual average temperature, rainfall, relative humidity and sunlight hours among the counties range from $16-18{ }^{\circ} \mathrm{C}$, 1000-1350 mm, 70-80\% and 1000-1400 h, respectively.

\section{Data collection Bacillary dysentery cases}

Daily reported data from January 1, 2009, to December 31, 2016 were obtained from Chongqing Municipal Center for Disease Control and Prevention. The data included each patient's gender, age, occupation, address and time of $\mathrm{BD}$ onset. According to the diagnostic criteria for bacterial dysentery and amoebic dysentery (WS 287-2008) issued by the Ministry of Health of the People's Republic of China, a clinically diagnosed $\mathrm{BD}$ case was defined based on following clinical features: fever, chills, abdominal pain, tenesmus, bloody or mucus stool, or stool containing N15/high-power field (HPF) leukocytes or purulent cells, and microscopically discernible red blood cells and phagocytic cells [16]. In 


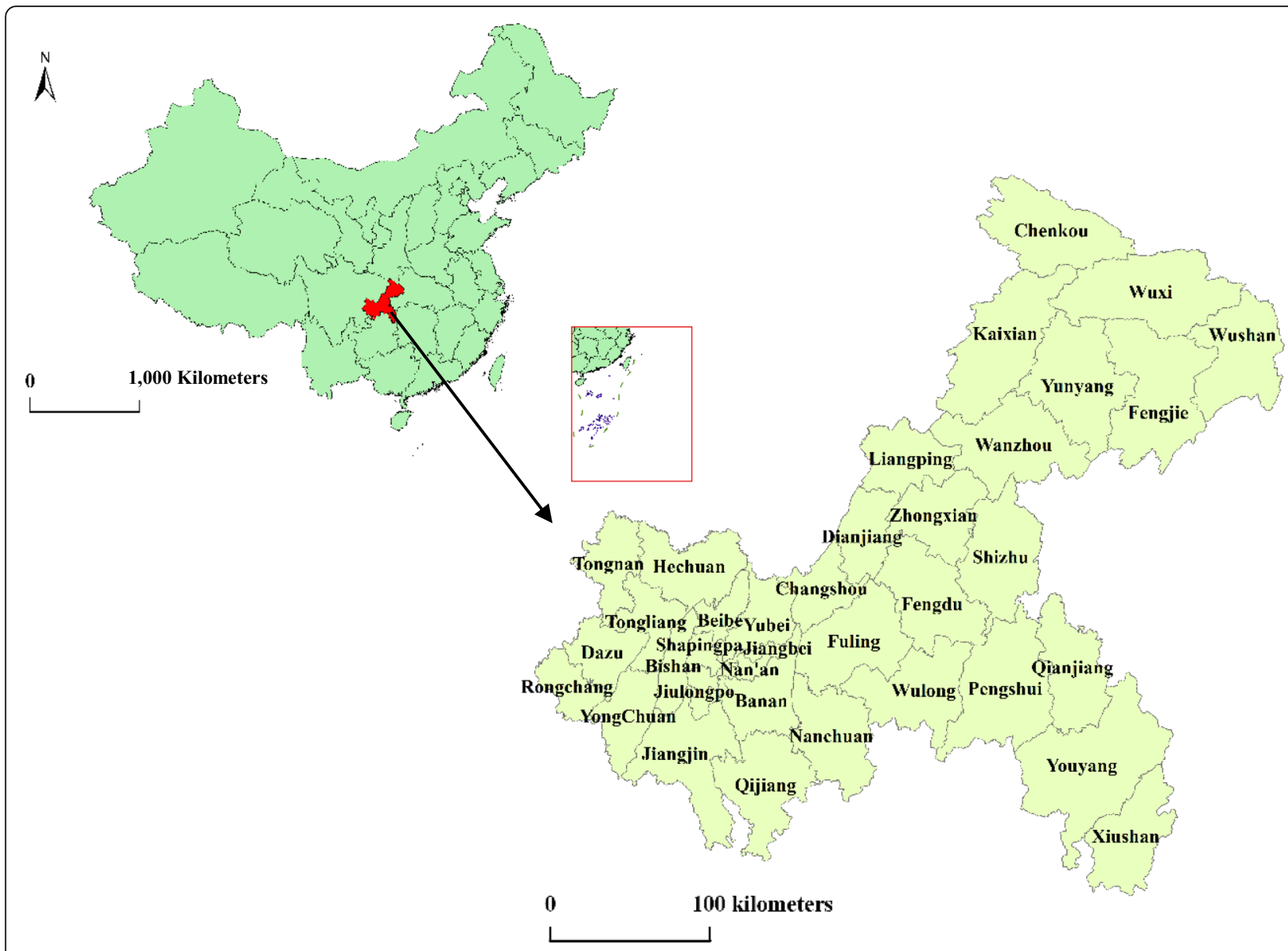

Fig. 1 Administrative division map in Chongqing at the county level

this study, BD cases included clinically diagnosed cases and confirmed cases through clinical diagnosis combined with pathogenic examination. All clinical and hospital doctors must report all clinically diagnosed and confirmed BD cases to the local centres for disease control and prevention (CDC) within $24 \mathrm{~h}$ through the Internet-based Chinese disease control and prevention information system.

\section{Meteorological data}

After summarising the previous studies, we found that air temperature, relative humidity, wind speed, sunshine, relative humidity and precipitation have an impact on BD incidence [11-14, 17]. To establish a prediction model with high prediction accuracy, ten meteorological factors on a daily basis (average air temperature $\left({ }^{\circ} \mathrm{C}\right.$ ), maximum air temperature $\left({ }^{\circ} \mathrm{C}\right)$, minimum air temperature $\left({ }^{\circ} \mathrm{C}\right)$, average air pressure $(\mathrm{hPa})$, water vapour pressure $(\mathrm{hPa})$, sunshine duration (h), relative humidity (\%), precipitation $(\mathrm{mm}), 2$ $\min$ wind speed $(\mathrm{m} / \mathrm{s})$ and $10-\min$ wind $\operatorname{speed}(\mathrm{m} / \mathrm{s}))$ were finally obtained from the Chongqing Meteorological Bureau. Chongqing Meteorological Bureau has set up 2200 general meteorological monitoring stations in the whole city and divided all general stations into 34 base stations according to their geographical location. In this study, the meteorological data of the whole city were obtained based on the average of the 34 base stations.

\section{Methods \\ Descriptive epidemiological analysis}

Descriptive epidemiological analysis was first used to analyse the epidemiological characteristics of $\mathrm{BD}$ from 2009 to 2016 in Chongqing to identify the distribution of BD. The results could help researchers develop more targeted public health interventions and provide a theoretical basis for disease prevention and control.

\section{Construction of predictive models}

Based on the correlation between meteorological factors and BD incidence, predictive models of BD incidence were established. Before the models were established, the data of $\mathrm{BD}$ cases and meteorological factors were collated monthly. Eighty percent of the data sets were randomly selected as models' training samples, while $20 \%$ of the data sets were used as models' test samples. In this study, the incidence of 
dysentery was used as the dependent variable, and the number of cases and meteorological factors in the 1-month period before the predicted time point were used as the independent variables in the models. The Boruta algorithm was used for the selection of the models' feature sets (i.e. variable sets) because the Boruta algorithm can select all of the feature sets related to the dependent variable instead of selecting a feature set that can make the model cost function the smallest for a specific model. Thus, the algorithm can help researchers understand the influencing factors of the dependent variable more comprehensively; this improves and increases the efficiency of feature selection and improves the accuracy of machine learning [18]. Then, according to the feature selection results and the hypothesis that the influence of meteorological factors on BD is complex and nonlinear, a support vector regression model (SVR) was selected to establish predictive models of BD incidence [19]. Finally, a genetic algorithm (GA) was applied to search for SVR model parameters (penalty parameter $C$, radial basis kernel function parameter $\gamma$ and threshold $\varepsilon$ of the $\varepsilon$-insensitive function) to improve the predictive ability of the SVR model [20].

\section{Statistical software}

The Boruta package in R-3.5.1 was used to implement the Boruta algorithm. Based on Matlab 2016a (MathWorks), the establishment of the epidemiological analysis and the predictive model of $\mathrm{BD}$ were completed. Additional files 2 and 3 show the core code and data set of the establishment of predictive model. The mean absolute percent error (MAPE), mean squared error (MSE) and squared correlation coefficient $\left(R^{2}\right)$ were selected as the indexes used to evaluate the precision of the models. The optimal predictive model was the one in which the values of MAPE and MSE were the smallest and the value of $R^{2}$ was the largest.

\section{Results \\ Epidemiological characteristics}

The total number of reported cases of BD in Chongqing from 2009 to 2016 was 68,855 ; the incidence declined from $36.312 / 100,000$ to $23.613 / 100,000$ and the average annual incidence was 29.394/100,000 during this period (Fig. 2). The top three incidences were among population subgroups aged 0-4, 5-9 and $\geq 65$ years; their incidences were 295.892/100,000; 24.938/100,000 and 22.524/100,000, respectively, which accounted for $52.879 \%, 4.627 \%$ and $8.925 \%$ of the total cases, respectively (Fig. 4 (left)). In the general population, the incidence in males (31.002/100, $000)$ was higher than that in females $(27.724 / 100,000)$ from 2009 to 2016, with a ratio of 1.118:1. With regard to the occupation distribution, pre-education children accounted for the most total cases (34,658 cases, 50.335\%), followed by farmers (8031 cases, $11.664 \%)$ and students (5592 cases, $8.121 \%)$. In terms of the temporal distribution, BD incidence showed obvious seasonality: it peaked in the period from May to October, with 45,131 cases, accounting for $65.545 \%$ of all cases (Figs. 3 and 4 (right)). In terms of the regional distribution, the cases were mainly concentrated in the metropolitan core area and the northeastern region of Chongqing (Fig. 5).

\section{The prediction models for morbidity based on meteorological factors}

The result of the feature set selected by the Boruta algorithm showed that meteorological factors, except the mean relative humidity and mean 2-min wind velocity, were deemed to be related to BD incidence (Fig. 6). Therefore, we used the monthly average of 8 important meteorological

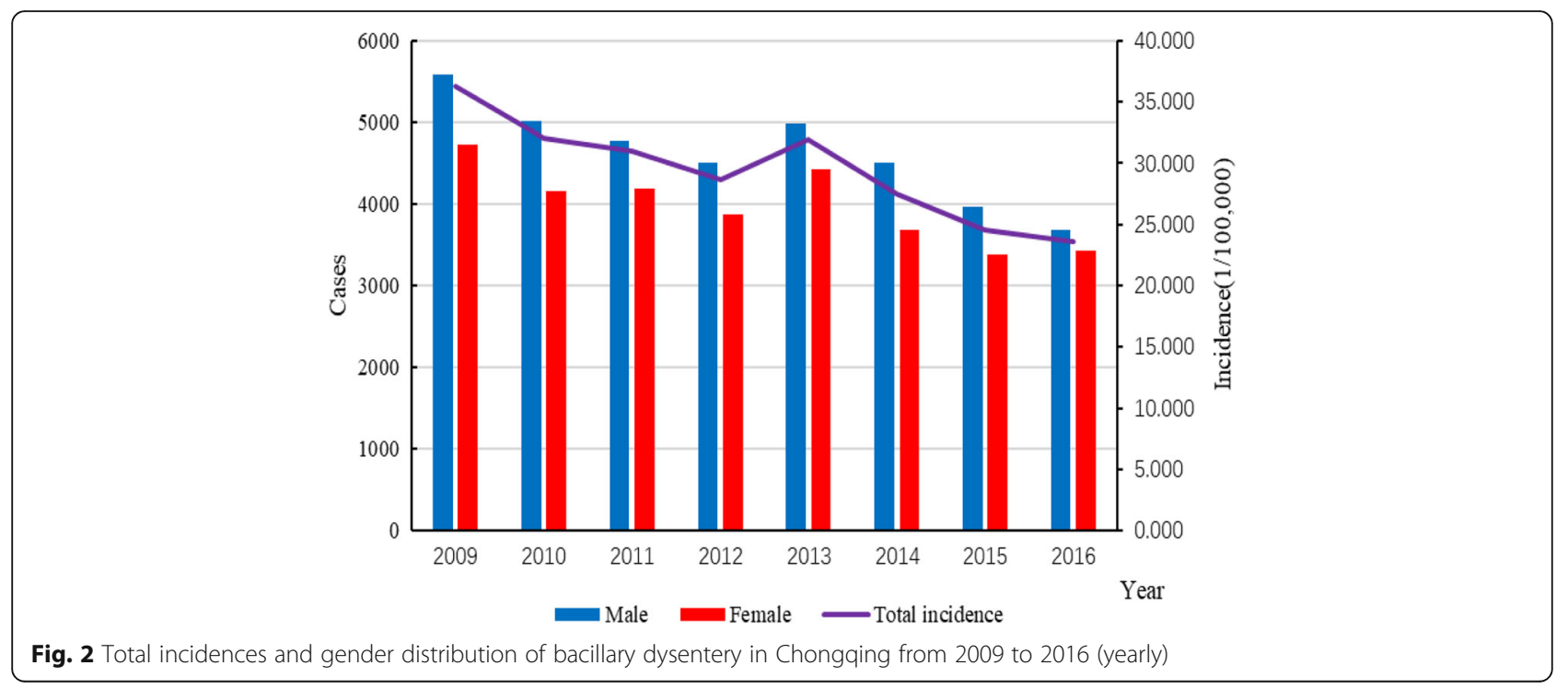




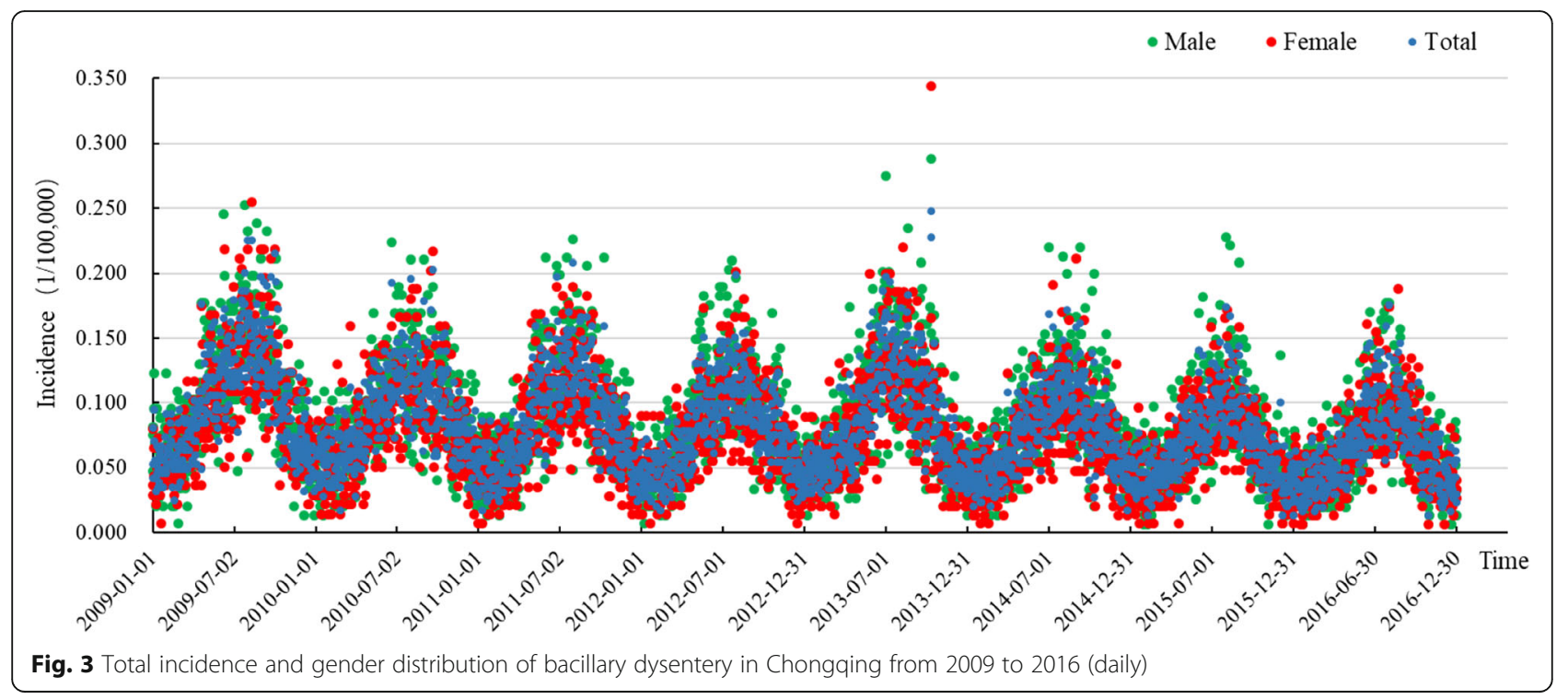

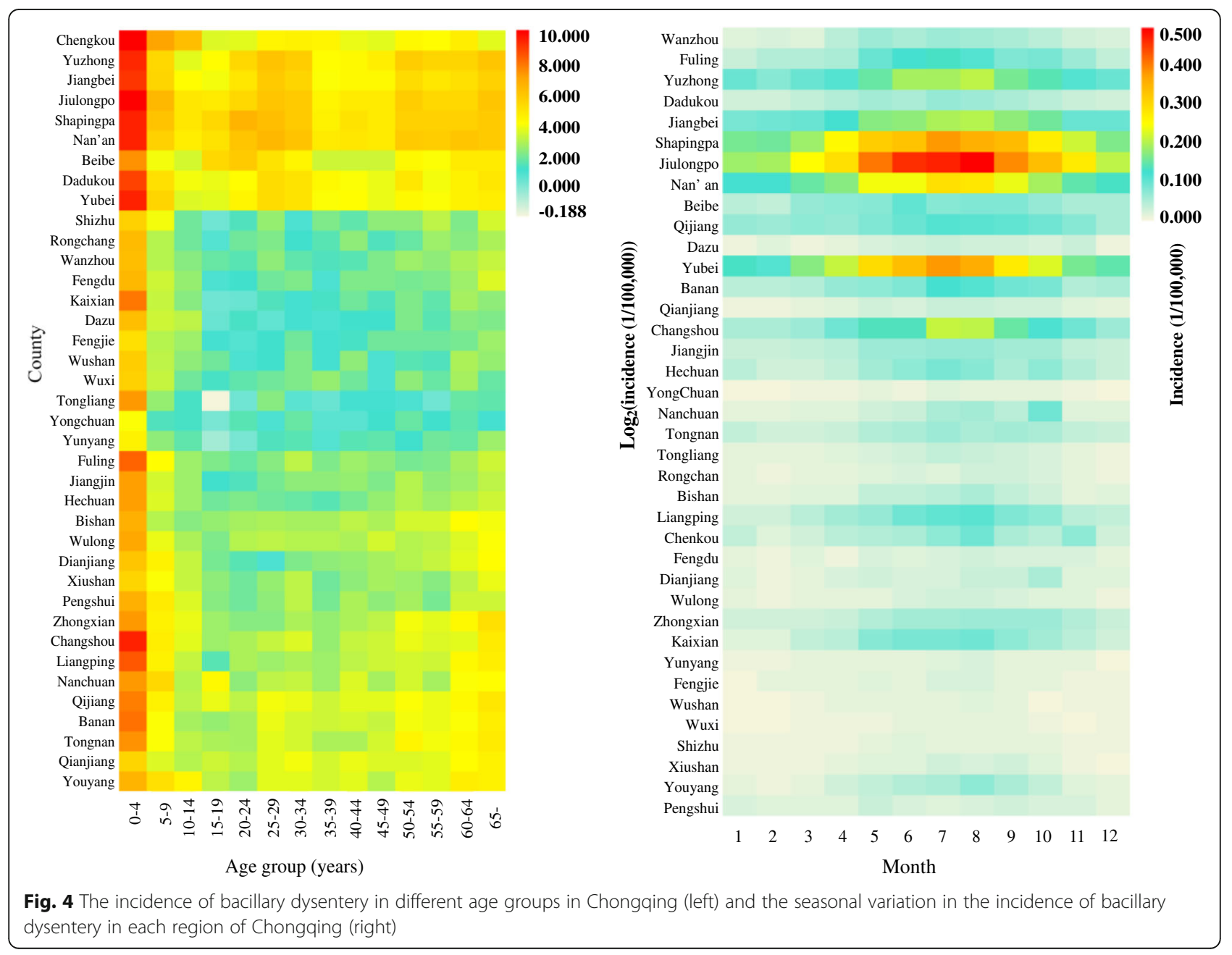




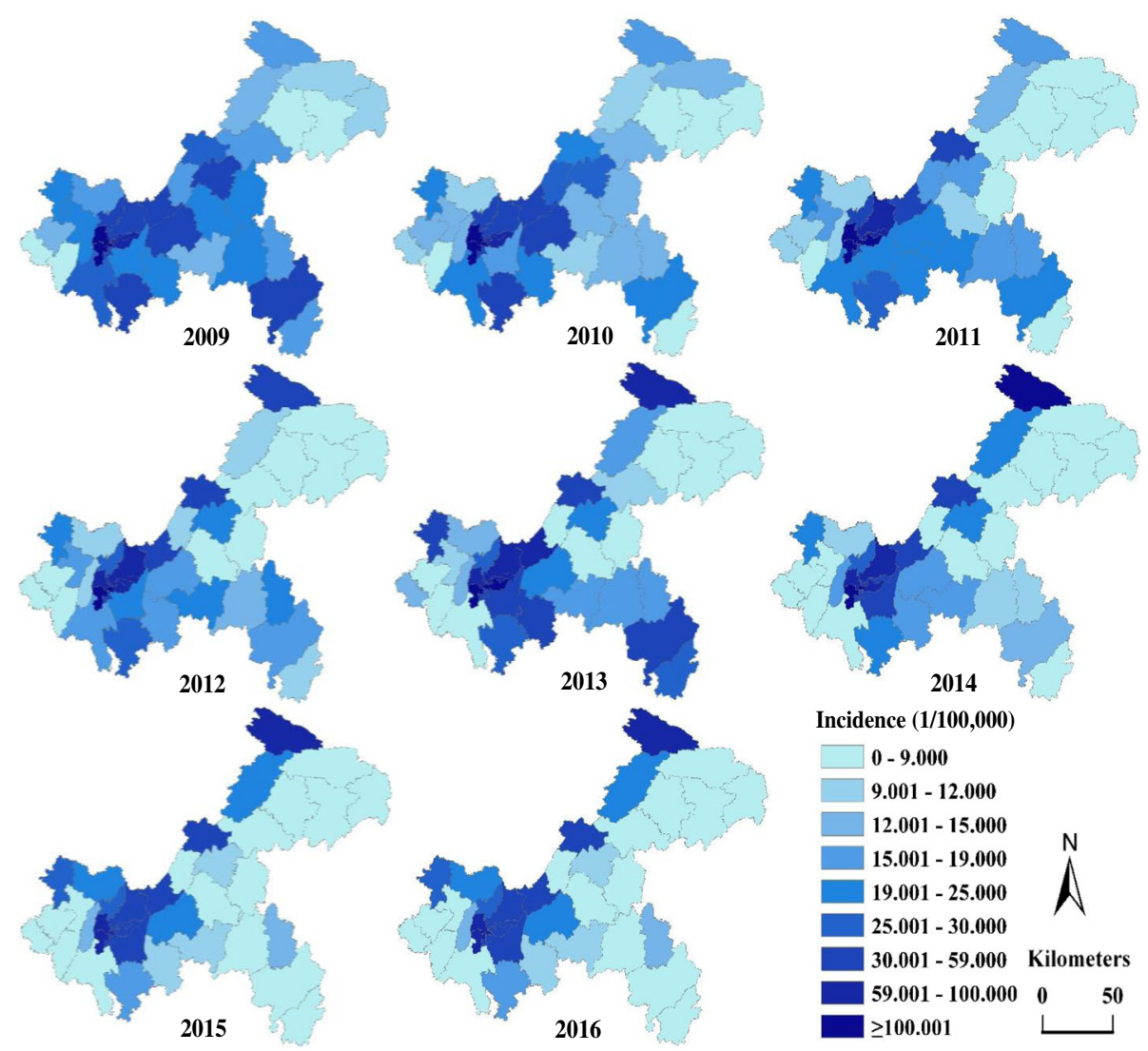

Fig. 5 Incidence maps of bacillary dysentery in Chongqing from 2009 to 2016

factors (average air temperature, maximum air temperature, minimum air temperature, average air pressure, water vapour pressure, sunshine duration, precipitation and 10min wind speed) and the number of BD cases as independent variables to establish the incidence prediction models.

\section{Establishment and effect evaluation of monthly $B D$ incidence prediction models}

The results show that the MSE, MAPE and $R^{2}$ values of the GA_SVR_MONTH model (the independent variables contained both BD incidence and meteorological factors) were $0.087,0.101$ and 0.922 , respectively. For comparison with the GA_SVR_MONTH model, the GA_SVR1_MONTH model that selected only the monthly number of patients as an independent variable was established, and its MSE, MAPE and $R^{2}$ were $0.214,0.172$ and 0.753 , respectively. Furthermore, the SVR_MONTH model without GA optimization (the independent variables were the number of patients and meteorological factors) and the SVR1_ MONTH model without GA optimization (the independent variable was only the number of patients) were established. Table 1 shows that the GA_SVR_MONTH model with the GA combined with meteorological factors was the most accurate. Compared with the GA_SVR1_MONTH,
SVR_MONTH and SVR1_MONTH models, the MSE decreased by $60 \%, 50 \%$ and $65 \%$, respectively; the MAPE decreased by $41 \%, 28 \%$ and $46 \%$, respectively; and the $R^{2}$ increased by $22 \%, 10 \%$ and $28 \%$, respectively. Figure 7 shows the fitting curves of GA_SVR_MONTH on the training set and test set.

In this study, all models were trained 10 times (each training sample was randomly selected); thus, the values of MSE, MAPE and $R^{2}$ in Table 1 are equal to the means of the corresponding predictive accuracy indicators of each of the 10 models.

\section{Discussion}

This study found that 68,855 BD cases were reported in Chongqing from 2009 to 2016, with an average annual incidence of 29.394/100,000. Although BD incidence in Chongqing showed a downward trend overall, it was still higher than the incidence in the same period in China $(20.28 / 100,000$ in $2009,15.29 / 100,000$ in $2012,11.24 /$ 100,000 in 2014) [21, 22], the USA in $2016(6.53 / 100$, 000), Zhejiang Province between 2004 and 2015 (4.25/ 100,000) and Sichuan Province between 2004 and 2014 $(22.12 / 100,000)[1,5,23]$, which highlights the serious situation regarding the prevention and control of BD in 


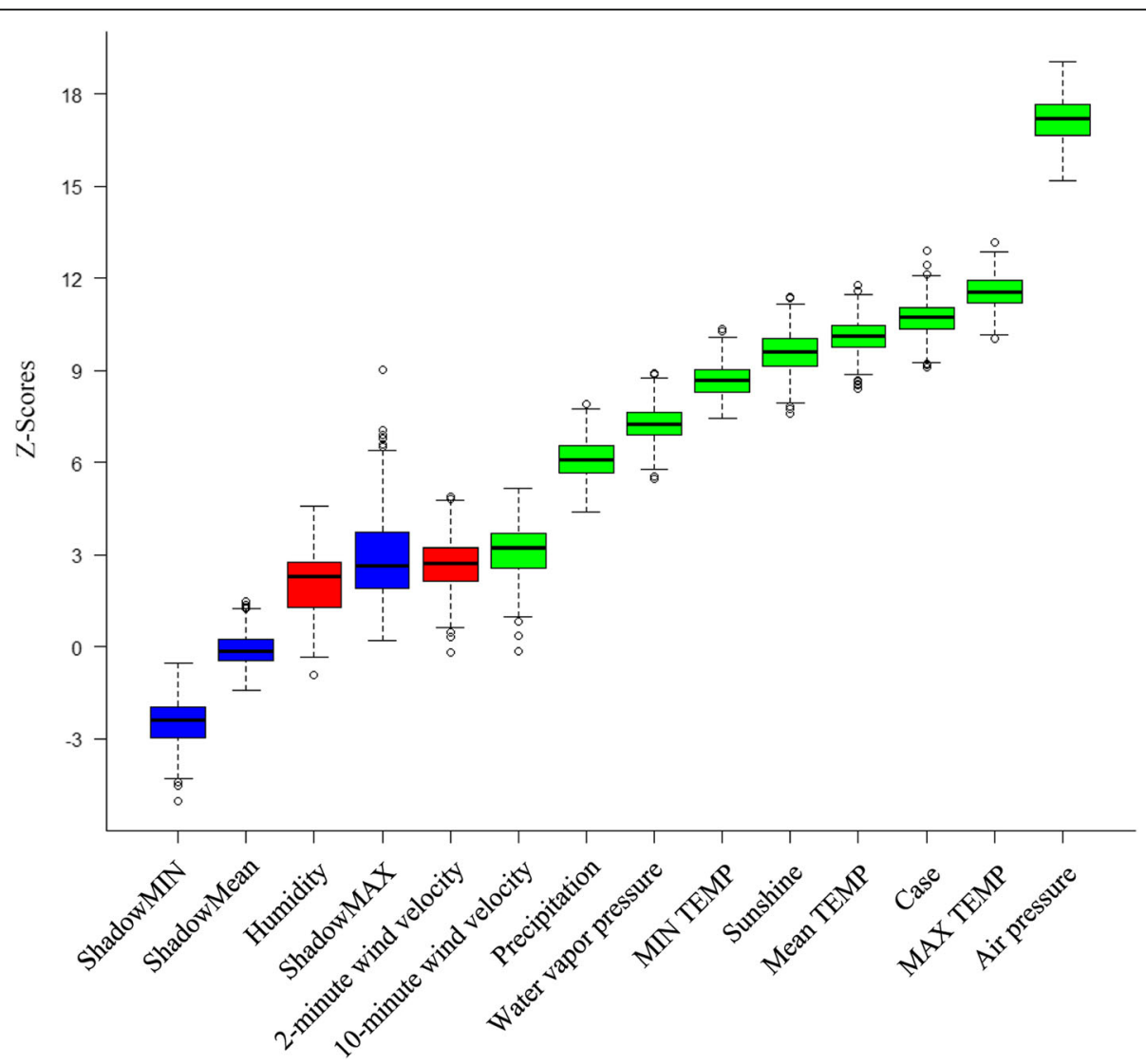

Fig. 6 Nine variables confirmed to be important: air pressure, max TEMP (temperature), mean TEMP, MIN TEMP, sunshine, water vapour pressure, precipitation, 10-min wind velocity and case. Note: The Z-score shows the importance of variables. The shadow variable is a random variable whose value is generated by permuting the original values across observations

Chongqing. Chongqing has a subtropical monsoon humid climate and covers an area of $824,000 \mathrm{~km}^{2}$, of which $78 \%$ is mountainous; it has sufficient precipitation, a warm temperature and a suitable climate for the growth and reproduction of Shigella, which may be one of the reasons BD incidence in Chongqing is higher than that in other areas. From 2009 to 2016, BD incidence in the population aged $0-4,5-9$ and $\geq 65$ years was relatively high in Chongqing. This is likely because the above population groups are mostly kindergarten children, primary school students, pre-education children and elderly people with weak immunity or poor hygiene habits. Furthermore, children in kindergarten institutions and schools are more concentrated; the environment is relatively closed, and it

Table 1 Comparison of the prediction accuracy of the monthly $\mathrm{BD}$ incidence prediction models

\begin{tabular}{llll}
\hline Model & MSE & MAPE & $R^{2}$ \\
\hline GA_SVR_MONTH & 0.087 & 0.101 & 0.922 \\
GA_SVR1_MONTH & 0.214 & 0.172 & 0.753 \\
SVR_MONTH & 0.172 & 0.141 & 0.840 \\
SVR1_MONTH & 0.250 & 0.186 & 0.721 \\
\hline
\end{tabular}

is very easy for pathogens to spread among individuals, which leads to outbreaks and relatively high prevalence rates [2, 24-26]. BD incidence was higher in men than in women in Chongqing, and the proportion of farmers was relatively large; this finding is consistent with other research results and may be attributed to that men are more likely to lack good health habits and to participate in group activities such as attending parties and dining out. Additionally, while the physical activity level of farmers is high, their working environments are frequently unsanitary $[1,5,8,21,27]$. This, coupled with a lack of knowledge of disease prevention and control, results in elevated incidences of BD among men and farmers. The period of high incidence of BD in Chongqing is mainly concentrated in May-October of each year. In summer and autumn, the temperature in Chongqing rises, the air pressure decreases, rainwater is abundant and flies and bacteria breed easily, which provides good conditions for the reproduction and transmission of Shigella [28-31]. In addition, a significant increase in temperature can not only change people's eating habits but also have adverse effects on body temperature and metabolism. For example, people tend to eat more raw and cold food when the temperature is high, 


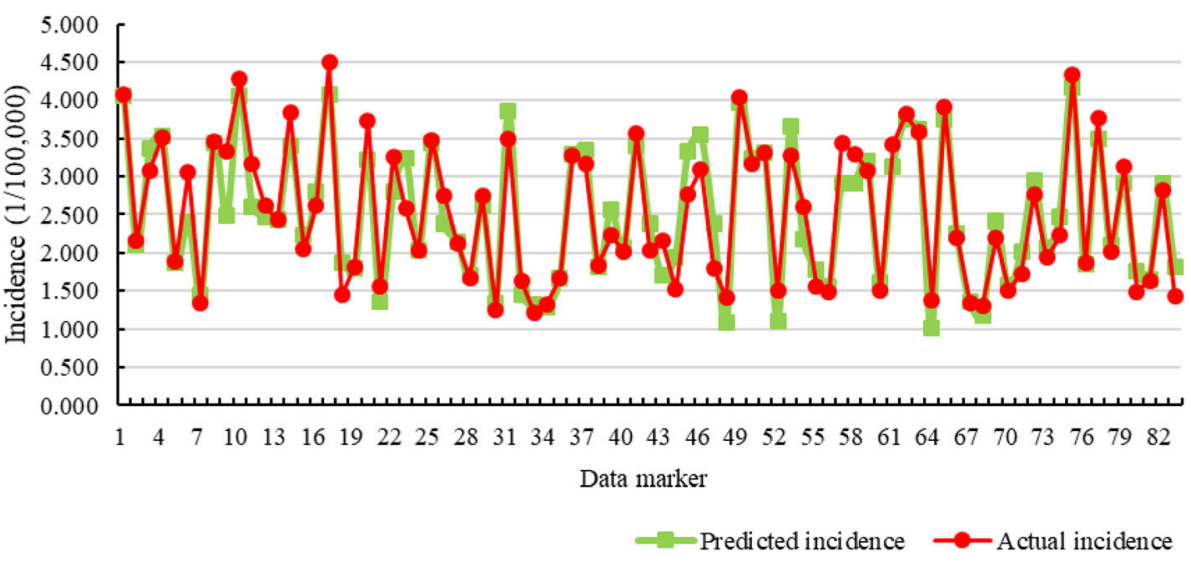

Fig. 7 The incidence predicted by the GA_SVR_MONTH model and the actual incidence: the 10th randomly selected training and test data set

and uncooked or cold food is more likely to contain pathogens. Additionally, heatstroke, which is caused by hightemperature exposure, could affect the immune system and make people more susceptible to infectious diseases [32]. Overall, relevant health sectors should take specific steps to prevent and control BD in key groups, high-incidence periods and areas in light of the epidemiological characteristics. For example, the high-incidence districts and counties can be included in the key prevention and control plan, and the prevention and control education regarding BD should be strengthened in key groups such as children, students and farmers to prevent the spread of dysentery.

The prediction of the incidence of infectious disease plays an important role in epidemic prevention and control as it provides decision-making support to relevant health sectors, enabling those sectors to formulate solutions and reduce the risk of an epidemic [33]. Therefore, this study has important significance in the field of disease research and the formulation of epidemic prevention and control strategies. In this paper, based on the correlations between meteorological factors and BD incidence, the Boruta algorithm and GA combined with the SVR model were used to establish predictive models of BD incidence. Our study fills a gap left by previous studies that did not fully consider the correlations between meteorological factors and BD incidence.

We chose the Boruta algorithm for feature selection because it is an efficient algorithm based on random forests. It can select all relevant variables to facilitate the establishment of a high-accuracy BD incidence predictive model. As Kursa et al. indicated, finding all relevant attributes, instead of only non-redundant ones, may be very useful. This is particularly necessary when one is interested in understanding mechanisms related to the subject of interest instead of merely building a black box predictive model [18]. Numerous diseases and environmental exposure-related studies have applied this algorithm for variable or feature selection [34-38]. The Boruta algorithm has been proven to be an efficient and stable feature selection algorithm. Although the feature set selected by the Boruta algorithm may contain highly collinear independent variables, such as minimum temperature, maximum temperature and mean temperature, multicollinearity of independent variables is not a problem for constructing prediction models [39]. Moreover, the BD prediction model we constructed shows satisfactory prediction accuracy, which also indicates that the Boruta algorithm is applicable to the selection of variables in this study.

The reason why we chose the SVR model to establish BD incidence prediction models is that the SVR model has good performance in solving regression problems. Sapankevyc et al. reviewed 66 studies that applied the SVR model to explore time series prediction and concluded that SVR has good performance in time series data prediction. Especially when the time series data have the characteristics of typical non-stationary and nonlinear data, the prediction effect of the SVR model is much better than that of other mathematical statistics and nonlinear analysis methods [40]. The reason could be that SVR has the global optimal solution when solving a model equation, while other methods (such as BP neural network) cannot guarantee the global minimum error value when optimising a network. In this paper, the MSE, MAPE and $R^{2}$ of the GA_SVR_MONTH model reached $0.087,0.101$ and 0.922 , respectively, which indicated high predictive accuracy of the model. By comparing the models established in this paper, the importance and rationality of meteorological factors as characteristic variables of the model used to predict $\mathrm{BD}$ incidence were confirmed. Furthermore, we used the same methods to establish predictive models of weekly BD incidence (GA SVR_WEEK). The MSE, MAPE and $R^{2}$ of the GA_SVR_ WEEK model, whose independent variables are BD cases and meteorological factors 4 weeks prior to the predictive week, reached $0.006,0.110$ and 0.888 , indicating satisfactory prediction accuracy (Additional file 1 Figures S1-S3). 
This study has several limitations. Although there are explicit diagnostic criteria and reporting management specifications for $\mathrm{BD}$, reporting bias may still exist in the data. Moreover, 1 month may not be an optimal predictive time. We will continue to explore a better predictive time based on the delayed effect of meteorological factors on BD. In addition, our study area is only one municipality directly under the central government in China, which leads to the limitation of extrapolating the conclusion to other regions with different socio-economic and meteorological conditions. In future studies, we will further optimise the settings and perfect the simulation to achieve a better model (Additional file 2: Figures S1-S3).

\section{Conclusion}

(1) The average annual incidence of BD in Chongqing from 2009 to 2016 was 29.394/100,000; this level was high, especially in the main urban areas and among the male and pre-educated-child populations.

(2) The GA_SVR_MONTH model, which was established based on meteorological factors, showed satisfactory predictive performance. In addition, meteorological factors were proven to obviously improve the accuracies of prediction models of $\mathrm{BD}$ incidence in this study. The findings in this study serve as an overview of BD in Chongqing and provide a useful predictive approach for the incidence of infectious disease, which can improve current interventions and public health planning.

\section{Supplementary information}

Supplementary information accompanies this paper at https://doi.org/10. 1186/s12199-019-0829-1.

Additional file 1: Figure S1-S3 Results about weekly models.

Additional file 2: Core code and data set of the establishment of predictive model.

Additional file 3: Core code and data set of the establishment of predictive model.

\section{Abbreviations}

BD: Bacillary dysentery; GA: Genetic algorithm; MAPE: Mean absolute percent error; MSE: Mean squared error; $R^{2}$ : Squared correlation coefficient;

SVR: Support vector regression model

\section{Acknowledgements}

Not applicable

\section{Authors' contributions}

Xun Liu and Qiuyu Meng designed the study, analysed the data and prepared the manuscript. They contributed equally to this work and should be considered co-first authors. Jiajia Xie, Dayong Xiao and Yi Wang revised the manuscript. Dan Deng designed the study and revised the manuscript. All authors read and approved the final manuscript

\section{Funding}

This study was part of the basic research and cutting-edge exploration project funded by the Scientific and Technological Commission of

Chongqing City (cstc2018jcyjAX0184).

\section{Availability of data and materials}

The data of cases analysed during the study are not publicly available due to regulations, but they can be obtained from the corresponding author on reasonable request. The core code and data set of models in the study are available in the supplemental materials.

\section{Ethics approval and consent to participate}

Not applicable

\section{Consent for publication}

Not applicable

\section{Competing interests}

The authors declare that they have no competing interests.

\section{Author details}

${ }^{1}$ School of Public Health and Management, Research Center for Medicine and Social Development, Innovation Center for Social Risk Governance in Health, Chongqing Medical University, Chongqing 400016, China.

${ }^{2}$ Department of Healthcare-associated Infection Control, The Second Affiliated Hospital of Military Medical University, Chongqing 400037, China. ${ }^{3}$ Institute for Prevention and Control of Endemic and Parasitic Diseases, Chongqing Center for Disease Control and Prevention, Chongqing 400042, China.

Received: 22 July 2019 Accepted: 12 November 2019

Published online: 28 December 2019

References

1. Yan C, Chen Y, Miao Z, Qin S, Gu H, Cai J. Spatiotemporal characteristics of bacillary dysentery from 2005 to 2017 in Zhejiang Province, China. Int J Environ Res Public Health. 2018;15(9):1826.

2. Hens N, Ayele GM, Goeyvaerts N, Aerts M, Mossong J, Edmunds JW, et al. Estimating the impact of school closure on social mixing behaviour and the transmission of close contact infections in eight European countries. BMC infectious diseases. 2009;9(1):187.

3. GBD Diarrhoeal Diseases Collaborators. Estimates of global, regional, and national morbidity, mortality, and aetiologies of diarrhoeal diseases: a systematic analysis for the Global Burden of Disease Study 2015. The Lancet Infectious diseases. 2017:17(9):909-48.

4. National Health and Family Planning Commission of the PRC. 2016 [Available from: http://www.nhfpc.gov.cn/jkj/s3578/201702/38ca5990f8a54 ddf9ca6308fec406157.shtml.

5. Ma Y, Zhang T, Liu L, Lv Q, Yin F. Spatio-temporal pattern and socioeconomic factors of bacillary dysentery at county level in Sichuan Province. China. Scientific Reports. 2015;5:15264.

6. Xu C, Li Y, Wang J, Xiao G. Spatial-temporal detection of risk factors for bacillary dysentery in Beijing, Tianjin and Hebei. China. Bmc Public Health 2017:17(1):743.

7. Liu X, Liu Z, Ding G, Jiang B. Projected burden of disease for bacillary dysentery due to flood events in Guangxi. China. Science of the Total Environment. 2017;601-602:1298-305

8. Liu Z-D, Li J, Zhang Y, Ding G-Y, Xu X, Gao L, et al. Distributed lag effects and vulnerable groups of floods on bacillary dysentery in Huaihua. China. Sci Rep. 2016;6(1):29456.

9. Mohammadi D, Naghshineh E, Sarsangi A, Sakhvidi MJZ. Environmental extreme temperature and daily preterm birth in Sabzevar, Iran: a time-series analysis. Environmental Health and Preventive Medicine. 2019;24(1):5.

10. Khan MS, Coulibaly S, Matsumoto T, Yano Y, Miura M, Nagasaka Y, et al. Association of airborne particles, protein, and endotoxin with emergency department visits for asthma in Kyoto. Japan. Environmental Health and Preventive Medicine. 2018;23(1):41.

11. Zhao Y, Zhu Y, Zhu Z, Qu B. Association between meteorological factors and bacillary dysentery incidence in Chaoyang city, China: an ecological study. BMJ Open. 2016;6(12):e013376. 
12. Xu C, Xiao G, Wang J, Zhang X, Liang J. Spatiotemporal risk of bacillary dysentery and sensitivity to meteorological factors in Hunan Province, China. International Journal of Environmental Research Public Health. 2018;15(1):47.

13. Liu Z, Liu Y, Zhang Y, Lao J, Zhang J, Wang H, et al. Effect of ambient temperature and its effect modifiers on bacillary dysentery in Jinan. China. Science of The Total Environment. 2019;650:2980-6.

14. Cheng J, Xie M, Zhao K, Wu J, Xu Z, Song J, et al. Impacts of ambient temperature on the burden of bacillary dysentery in urban and rural Hefei. China. Epidemiology Infection. 2017;145(8):1567-76.

15. Zhang N, Zeng W, Fang Y. Analysis of the reporting and surveillance result of bacillary dysentery in Nan'an district of Chongqing in 2011 [in Chinese]. Modern Preventive Medicine. 2013;40(19):3694-3695 + 3699.

16. Ministry of Health of the People's Republic of China . Diagnostic criteria for bacterial dysentery and amoebic dysentery [in Chinese]. Available from: http://www.nhc.gov.cn/wjw/s9491/200802/39040/files/9c939b0b5de04a14 be37e02421adc661.pdf.

17. Du Z, Zhang J, Lu JX, Lu LP. Association between distribution of bacillary dysentery and meteorological factors in Beijing, 2004-2015 [in Chinese]. Zhonghua liuxingbingxue zazhi. 2018;39(5):656-60.

18. Kursa MB, Rudnicki WR. Feature selection with the Boruta Package. Journal of Statistical Software. 2010;36(11):1-13.

19. Cortes C, Vapnik V. Support-vector networks. Machine Learning. 1995;20(3): 273-97.

20. Holland JH. Genetic Algorithms. Scientific American. 1992;267(1):66-72.

21. Chang Z, Zhang J, Ran L, Sun J, Liu F, Luo L, et al. The changing epidemiology of bacillary dysentery and characteristics of antimicrobial resistance of Shigella isolated in China from 2004-2014. BMC Infect Dis. 2016;16(1):685.

22. Chang Z, Sun Q, Pei Y. Bacillary dysentery characteristics and surveillance analysis of bacillary dysentery in mainland in 2012. Disease surveillance. 2014;29(7):528-32.

23. Centers for Disease Control and Prevention 2018 [cited 2018]. Available from: https://wonder.cdc.gov/nndss/static/2016/annual/2016-table1.htm

24. Chen Y, Badaruddin H, Lee VJ, Cutter J, Cook AR. The effect of school closure on hand, foot, and mouth disease transmission in Singapore: a modeling approach. The American journal of tropical medicine and hygiene. 2018;99(6):1625-32.

25. Prem K, Cook AR, Jit M. Projecting social contact matrices in 152 countries using contact surveys and demographic data. PLoS computational biology. 2017;13(9):e1005697.

26. Kotloff KL, Riddle MS, Platts-Mills JA, Pavlinac P, Zaidi AKM. Shigellosis. Lancet (London, England). 2018;391(10122):801-12.

27. Zhang J, Mahemuti M, Xia YD, Mutalifu M, Muheyati M, Li F, et al. Epidemiology and etiology of bacillary dysentery in Xinjiang Uigur Autonomous Region, 2004-2014 [in Chinese]. Zhonghua liuxingbingxue zazhi. 2016;37(11):1526-30.

28. Das JK, Hadi YB, Salam RA, Hoda M, Lassi ZS, Bhutta ZA. Fly control to prevent diarrhoea in children. The Cochrane database of systematic reviews. 2018;12:Cd011654

29. Xu Z, Liu Y, Ma Z, Toloo G, Hu W, Tong S. Assessment of the temperature effect on childhood diarrhea using satellite imagery. Scientific Reports. 2014; 4:5389.

30. Farag TH, Faruque AS, Wu Y, Das SK, Hossain A, Ahmed S, et al. Housefly population density correlates with shigellosis among children in Mirzapur, Bangladesh: a time series analysis. PLoS Negl Trop Dis. 2013;7(6):e2280

31. Viswanathan VK. Shigella takes the temperature. Gut microbes. 2013; 4(4):267-8.

32. Kuo-Cheng L, Shih-Hua L, Pauling C, Weng-Sheng T, Yuh-Feng L. Correlation of neutrophil phagocytosis and lymphocyte adhesion molecules in exertional heat stroke. American Journal of the Medical Sciences. 2004; 327(2):68-72.

33. Guan $P, W u$ W, Huang $D$. Trends of reported human brucellosis cases in mainland China from 2007 to 2017: an exponential smoothing time series analysis. Environmental Health and Preventive Medicine. 2018;23(1):23.

34. Singla M, Kar M, Sethi T, Kabra SK, Lodha R, Chandele A, et al. Immune response to dengue virus infection in pediatric patients in New Delhi, IndiaAssociation of Viremia, Inflammatory Mediators and Monocytes with Disease Severity. Plos Neglected Tropical Diseases. 2016;10(3):e0004497.

35. Yang L, Li L, Ma J, Yang S, Zou C, Yu X. miRNA and mRNA integration network construction reveals novel key regulators in left-sided and right- sided colon adenocarcinoma. Biomed Research International. 2019;2019: 7149296.

36. Lin J, Jiang A, Ling M, Mo Y, Li M, Zhao J. Prediction of neurologic deterioration based on support vector machine algorithms and serum osmolarity equations. Brain and Behavior. 2018;8(7):e01023.

37. Lu HL, Zhao MS, Liu BY, Zhang P, Lu LM. Predictive mapping of soil PH in Anhui Province based on Boruta-support vector regression [in Chinese]. Geography and Geo-Information Science. 2019;35(5):66-72.

38. Zhou Y, Zuo Z, Xu F, Wang Y. Origin identification of Panax notoginseng by multi-sensor information fusion strategy of infrared spectra combined with random forest. Spectrochimica acta Part A. Molecular and biomolecular spectroscopy. 2019;226:117619.

39. Makridakis SG, Wheelwright SC, Hyndman RJ. Forecasting: methods and applications. 3rd ed. New York: Wiley; 1998. p. 288.

40. Sapankevych NI, Sankar R. Time series prediction using support vector machines: a survey. IEEE Computational Intelligence Magazine. 2009; 4(2):24-38.

\section{Publisher's Note}

Springer Nature remains neutral with regard to jurisdictional claims in published maps and institutional affiliations.

\section{Ready to submit your research? Choose BMC and benefit from:}

- fast, convenient online submission

- thorough peer review by experienced researchers in your field

- rapid publication on acceptance

- support for research data, including large and complex data types

- gold Open Access which fosters wider collaboration and increased citations

- maximum visibility for your research: over $100 \mathrm{M}$ website views per year

At BMC, research is always in progress.

Learn more biomedcentral.com/submissions 